DOI: https://doi.org/10.18485/bells.2016.8.4

UDC: 811.111'366.543

\author{
Jelena Vujić ${ }^{*}$ \\ Nenad Miladinović ${ }^{* *}$ \\ University of Belgrade \\ Faculty of Philology \\ Serbia
}

\title{
GENITIVE CONSTRUCTIONS AS CONSTRUCTIONAL IDIOMS IN ENGLISH
}

\begin{abstract}
This paper is a study of formal, semantic and pragmatic properties of the idiomatic structure a N1 of a N2 with the meaning "N2 resembles N1". We analyze the given form within the framework of Construction Morphology which we believe is the most viable analytic tool for constructional idioms, the phenomena that display strong phrase-like and word-like properties, without losing the generalizations that exist between the two.
\end{abstract}

Key words: genitive, construction, construct, constructional idiom, Construction Morphology.

\section{Introduction}

Lexicon is a set, a repository of all simple and complex words that are idiosyncratic or conventionalized. Even pieces of syntactic structure can be listed in the lexicon with associated meanings (Jackendoff 2008:

\footnotetext{
E-mail address: jelenajvujic@gmail.com

** E-mail address: nenad_miladinovic@yahoo.com
} 
15). Lexicon, therefore, apart from simple words or one-word lexemes must specify all syntactically complex words, also known as multi-word expressions (MWE). MWEs display higher or lower degree of idiosyncratic meaning. Such expressions are typically constructs with more or less unpredictable properties that have to be memorized and learned by the speaker. They vary in their size and complexity. As for their meaning, as much as they may be regular in their form representing sequences of words, the meaning of idiomatic constructs often is not atomic/ compositional in nature (at least not entirely). Rather they differ in their flexibility and degree of their compositionality. However, the fact that they are complex means that they are constructed, formed and created from at least two elements, having a certain structure. Thus they create a construction of this sort or another. These are meaningful constructions of the language.

In this paper we show how the notion construction can be very useful in describing and analyzing syntactic word combinations which display both word-like and phrase like properties and can serve as lexical units. In order to highlight the dual nature of certain phrasal lexical units and to show how the only way to account for a strong lexicon-syntax interaction and interrelatedness is via constructionist approach we will focus on formal and semantic properties of a particular type of of-genitive construction. As we will show the construction in question presents a cross-language phenomenon.

As lexical units can be both word level constructions and phrase level constructions it implies a much tighter relation of syntax, morphology and the lexicon. From the need for a theory which will provide the most suitable framework within which mutual commonalities and differences of syntax, morphology and lexicon will be accounted for the theory of Construction Morphology (CM) has arisen. CM does not see syntax and morphology as two separate language and lexicon components but rather as complementary segments.

Constructions, are pairings of meaning and form (and function). Traditionally speaking constructions lie in the domain of syntax rather than that of morphology. However, with advances of Construction Grammar the perception of construction has changed. In modern linguistic science it is considered to be an abstract linguistic unit which can be found on all linguistic levels, including morphological level. 
Jelena Vujić, Nenad Miladinović: Genitive Constructions as Constructional Idiom in English

Constructions resemble mathematical formulae in their formal structure which means that certain structures are possible while others are not, or at least not with the same reference. This is illustrated in the following examples of Determinative construction (1), Modifying construction (2) and genitive construction (3).

(1a) the underprivileged, a lion, my only child

(1b) * underprivileged the, * lion a, *only child my

(2a) hard-working man, women in black, love that stunned the world

(2b) * man hard-working, * in black women, *that stunned the world love

(3a) A man of courage, a portrait of an artist

(3b) Courage of a man, ? an artist of a portrait

Examples (1), (2a) and (3a-b) can be schematically represented as (4a-c).

(4a) $[[\mathrm{x}] \operatorname{det}[\mathrm{y}] \mathrm{N}]$ for Determinative Construction

(4b) $[[\mathrm{x}] \operatorname{adj}[\mathrm{y}] \mathrm{N}]$ or $[[\mathrm{x}] \mathrm{N}[\mathrm{z}] \mathrm{PP}]$ for Modifying construction

(4c) $\left.\left[[\mathrm{X}]_{\mathrm{Ni}}\left[[\mathrm{of}]_{\mathrm{P}}\left[[\text { the }]_{\mathrm{det}}[\mathrm{x}]_{\mathrm{Nj}}\right]_{\mathrm{NP}}\right]_{\mathrm{PP}}\right]\right]_{\mathrm{NP}}$ for Of-genitive Construction

Formal structures such as those represented in (4a-c) typically trigger certain (general) semantic interpretation. For example, modifying construction triggers the interpretation " $\mathrm{X}$ modifies, describes $\mathrm{Y}$ by attributing certain qualities to it", while a prototypical meaning of of-constructions is that of possession. While the slots in (4a-c) are distributionally fixed and categorically specified, there are numerous individual instantiations of these two types of constructions as given in (1a) (2a) and (3a-b), which are referred to as constructs. They represent concrete realizations of the patterns given above.

Clearly all given constructions are syntactic in their nature and meaning. The distribution of their elements and their category are restricted and fixed, whereas the choice of particular words-lexemes, which represent variables, is free. By varying the variables various constructs get formed. Constructs enable us to get concrete, particular semantic interpretations of (general) abstract constructions. For example, in the phrase deep sea $\mathrm{Y}$ is the concrete noun with particular meaning sea ("a large body of water") which is modified, qualified by $\mathrm{X}$, the adjective deep (with the meaning "having a quality of great depth"). 
In (4a-c) we have given constructional schema for syntactic structures with more or less grammatical meaning (denoting grammatical relations). However, a large number of syntactic patterns are lexical in their semantics, which means that they not only have a rather fixed syntactic structure but also the choice of lexemes may be quite restricted or even totally fixed. When they are completely fixed both formally and lexically with specific semantic content, which may be accompanied with rather low productivity of the pattern (but not necessarily), they are referred to as idiomatic phrasal units (idioms).

English lexicon is full of complex lexical units with a higher or lower level of idiosyncrasy and with more or less fixed choice (and distribution) of words for a particular construction. They are referred to as multi-wordunits. When dealing with such complex words which are phrasal in nature, the notion of construction comes particularly interesting and useful. Like all constructions, multi-word-units represent syntactic patterns in which certain formal properties correlate with certain semantics. They, too, can vary in the degree of lexical and syntactic fixedness.

In some cases new lexical units with syntactic properties may reflect the syntactically productive pattern which is typically interpreted to carry syntactic meaning. Such are patterns for constructions with strong grammatical meaning and relevance such as of-genitive construction (5a). In (5a) a syntactic pattern for Norman genitive is used for naming purposes to denote titles or particular concepts etc, which is not the case with (5b).

(5a) The Queen of England, the Duchess of Cambridge, the ace of spades, the change of heart.

(5b) I did not possess a painting of an artist.

Both examples (5a -b) can be formally represented by a constructional schema as follows:

(6) $\left.\left[[\mathrm{X}]_{\mathrm{Ni}}\left[[\mathrm{of}]_{\mathrm{P}}\left[[\text { the }]_{\mathrm{det}}[\mathrm{x}]_{\mathrm{Nj}}\right]_{\mathrm{NP}}\right]_{\mathrm{PP}}\right]\right]_{\mathrm{NP}} \leftrightarrow\left[\text { SEM }_{\mathrm{j}} \text { possesses SEM }\right]_{\mathrm{NP}}$

In such cases we speak of open syntactic constructions which reflect only distributional and categorial fixedness. In other words, the combination in the structure det $+\mathrm{N}+$ of $+\mathrm{N}$ is fixed and as for categorial fixedness it means that each slot can be filled only with a particular lexical category. Speakers freely choose the lexical units to fill the slots, also known as variables, as long as they follow the restrictions specified in the constructional schema. Thus, they compute new constructs according to the stored schema pattern and the interpretation is always the same. For example, the combination 
Jelena Vujić, Nenad Miladinović: Genitive Constructions as Constructional Idiom in English

Adj. (X ) + N (Y) always instigates the meaning: $Y$ has the quality $X$. Typically, all instances of syntactic constructions of the type given in (1a), (2a) and (3a-b) are compositional in their meaning.

Syntactic constructions with idiomatic status, on the other hand, reflect very high level of syntactic and lexical fixedness; consequently, they need to be stored in our lexicon. They are not computed by the speakers but simply retrieved as prefabricated chunks of language.

However, there are phrases that follow a completely regular pattern and whose meaning may be compositional, though it is highly conventionalized as those given in (7).

(7) a hell of a trip, a nightmare of a journey, an angel of a woman.

Such expressions follow seemingly prototypical syntactic patterns reflecting general structures of particular syntactic structures while instigating specific lexical (idiomatic) meaning. Our examples follow the pattern of Norman genitive construction (of-genitive construction). They typically display partial fixedness in the domain of their structure and the distribution of their elements. Their meaning is either only partially compositional (which is the case with our examples) or not compositional at all. This means that their meaning cannot be (entirely) inferred based on the atomic meanings of their constituents but it has to be stored. In other words, the semantics of lexemes constituting particular constructs participates in the meaning of the entire expression but they have to be interpreted in certain order and with certain reference. Quite often due to their lexico-semantic and syntactic properties they are examples of the lexical units in which morphology, syntax and lexicon interact and overlap.

Formally, expressions like those are structurally nearly identical to those presented in (5a-b). Yet, their reading is different and can be represented with the constructional schema as follows:

$$
\left[[\mathrm{x}]_{\mathrm{Ni}}\left[[\mathrm{of}]_{\mathrm{P}}\left[[\mathrm{a}]_{\text {Det }}[\mathrm{x}]_{\mathrm{Nj}}\right]_{\mathrm{NP}}\right]_{\mathrm{Pp}}\right]_{\mathrm{Nk} \leftrightarrow}\left[\mathrm{SEM}_{\mathrm{j}} \text { with } \text { SEM }_{\mathrm{i}} \text {-like property }\right]_{\mathrm{k}}
$$

From the constructional schema (8) we see that such phrases exemplify the general structure of English NPs with an N as a head, preceded by the primary determiner and followed by a prepositional phrase. Semantically, however it is the noun within the prepositional phrase (N2), which is positioned as a prepositional complement, that acts as head, while N1 acts as its modifier. As head, N2 also governs further syntactic pronominal agreement.

(9) That giant of a woman brought all her kids with her. 
Despite its structural similarity to the possessive of-genitive construction, there is no doubt that this type of syntactic phrase is the type that has to be stored in the lexicon as highly conventionalized. As a result, the schema like (8) is to be classified as a constructional idiom (Booij 2010: 13). In other words, it is a type of an idiom in which not all positions are lexically fixed. In structure (8) the nominal positions are open and in principle, can be filled with any noun. However, the prepositional and determiner positions are specified. The schema of this constructional idiom represents a productive pattern, which means that the list of expressions is not fixed. The constructs of this type can be extended though the extension is not unlimited. The key factor for extension are the semantic restrictions that this construction as a whole imposes on its variable constituents. The implication of this form on the extension possibilities will be discussed later in this paper after we have given an overview of its formal, structural and lexico-semantic properties (frame).

\section{Formal properties of of-genitive construction and constructional idioms of the type a monster of a truck}

In the previous part of this paper we have seen that the constructional idiom of the type a monster of a truck in formal aspect follows the pattern of of-genitive construction, the one that is prototypically interpreted as conveying a possessive relationship between $\mathrm{N} 1$ and N2. The possible extensions of this prototypical possessive meaning which are all seen as regular compositional grammatical meanings are given below.

\section{a) Semantic interpretation of of-genitive construction}

As noted before, the pattern of of-genitive construction is prototypically interpreted as conveying a possessive relationship between $\mathrm{N} 1$ and N2.

However, even a prototypical meaning of of- genitive construction with the schema $\left.\left[[\mathrm{X}]_{\mathrm{Ni}}\left[[\mathrm{of}]_{\mathrm{P}}\left[[\text { the }]_{\text {det }}[\mathrm{x}]_{\mathrm{Nj}}\right]_{\mathrm{NP}}\right]_{\mathrm{PP}}\right]\right]_{\mathrm{NP}} \leftrightarrow \rightarrow\left[\mathrm{SEM}_{\mathrm{j}} \text { possesses SEM }\right]_{\mathrm{NP}}$ may have multiple meanings. Although the meanings are numerous, they all directly or indirectly originate from possessive relation that exists between $\mathrm{N} 1$ (h) as a possessum and N2 (d) as a possessor. Different instances of ofgenitive construction given below represent a higher or lower degree of extension of the prototypical meaning (and schema) - possessive meaning. However, we must point out that the extension of meaning (and schema) 
Jelena Vujić, Nenad Miladinović: Genitive Constructions as Constructional Idiom in English

does not in any way affect the structure and form of this constructional pattern.

Of-genitive construction triggers the multitude of interpretations. According to Miladinović (Miladinović 2015: 230-234) ${ }^{1}$ the possible relations that is established by this construction between N1 and N2 include among others body-part, kin, subordinate, superior relations as well as the relations indicating ownership, cause and result, content, temporal relation, measures etc. In total, he gives some 36 different relations that are depicted by the pattern $\left.\left[[\mathrm{X}]_{\mathrm{Ni}}\left[[\mathrm{of}]_{\mathrm{P}}\left[[\text { the }]_{\text {det }}[\mathrm{x}]_{\mathrm{Nj}}\right]_{\mathrm{NP}}\right]_{\mathrm{PP}}\right]\right]_{\mathrm{NP}} \leftrightarrow\left[_{\text {SEM }} \text { possesses SEM }\right]_{\mathrm{NP}}$

\section{b) Formal properties of constructional idiom a monster of a truck}

The constructional idiom of this type is structurally identical to of- genitive construction. Syntactic distribution of this construction's constituents is different, which results in its different semantics. In of-genitive construction $\mathrm{N} 1$ is postmodified by prepositional of-phrase (of $+\mathrm{N} 2$ ). Actually, N2 as a postmodifier is added extra semantic content (denoting the genitive) by being preceded by OF. In other words, it is the noun N2, formally marked for genitive that modifies N1. All this is explicitly encoded in the constructional schema of this pattern given in (8).

On the other hand, the constructional idiom a monster of a truck actually displays the syntactic relation of a premodifier $+\mathrm{H}$. Many scholars see this pattern as two NPs in apposition with N2 being the head. N2 is formally marked for genitive while $\mathrm{N} 1$ is a dependant constituent of the construction. The fact that the head is prepositionally marked for genitive is atypical for English.

Proof that the particle of actually stands for genitive here can be found in a number of different languages that recognize this pattern with exactly the same syntactic and semantic interpretation. Such patterns are found in Dutch, French, Spanish, Italian, German and Serbian among other languages.

(10) Dutch: een schat van een kind (a sweetheart of a child) German: ein Teufel von einem Mann (a devil of a man)

Spanish: esa mierda de libro (that shit of book)

French: ton phenomene de fille (your phenomenon of daughter) Serbian: beda od čoveka, lutka od deteta, krš od automobila

\footnotetext{
1 Miladinović derives his sub-classification of of-genitive meanings from the classification given by Huddlestone and Pullum for the Saxon genitive (Huddleston \& Pullum 2002: 473). For a detailed list of all of-genitive meanings see Miladinović (2015: 230-234).
} 
They all share the same common property that the $\mathrm{H}$ is the latter noun and that it is formally marked for genitive both inflectionally (as in Serbian) and syntactically-prepositionally (as in French, Dutch) ${ }^{2}$. The prepositions used in the cases of inflectional languages (Serbian) are typically those occurring with genitive (only). In English it is the preposition of and in Serbian the preposition od as in beda od coveka, lutka od deteta. Thus, such prepositions do not trigger any meaning other than genitive. It would be interesting to delve into the meaning of genitive here. We hope it to be a matter tackled in subsequent papers and research. There is a rather problematic issue of the existing genitive meanings specified earlier since in this pattern, it is the semantic head of the phrase that is marked for genitive. Such "genitive head" is modified and qualified. Therefore, the qualifying and descriptive meaning of the genitive cannot not refer to the meaning of the entire head as it is the head that is modified. The meaning could be interpreted as " an instance, one particular aspect of the genitive noun N2 is N1 as a premodifier". In the example a monster of a truck it is the truck which has all properties prototypically associated with trucks which are additionally accompanied with some secondary properties that emphasize its huge size. Secondary properties expressed by N1 usually become (either truly or metaphorically) so dominant that they overshadow the prototypical properties of $\mathrm{N} 2$.

It is a very open frame which displays morphological nature in the respect that new items can be created when thought desirable thus making nonce-formations or portmanteau formations (e.g. 'a ponytail of a waterfall'). On the other hand, this is the frame whose certain instances become highly colloquial (e.g. 'a gem of a ...' '... of a man') or strongly lexicalized ('a whale of a time'). The frame has "long formed part of the syntactic description of English, but it has not been possible to describe it in much detail because of the paucity of authentic examples" (Coffey 2009).

A certain level of fixedness undoubtedly present in English genitive construction of the type NP1 + of + NP2 emphasizes the lexical side of its nature. Such fixedness is manifested in the following aspects:

a) (almost mandatory) presence of the indefinite article $a(n)$ in the positions preceding both $\mathrm{N} 1$ and N2;

b) mandatory preposition of in medial position between NP1 and NP2;

c) free, open slots for lexical variables of N1 and N2.

${ }_{2}$ In German this construction triggers the same meaning as in other languages but it is associated with dative rather than genitive. 
Jelena Vujić, Nenad Miladinović: Genitive Constructions as Constructional Idiom in English

Even at first sight, it is clear that what distinguishes prototypical of-genitive construction and this constructional idiom is that the latter requires presence of a determiner, preferably and prototypically indefinite article $a^{3}$. Variable levels of fixedness of this construction are illustrated by the fact that other determiners may occupy initial position in NP1. In his research on grammatical and lexical frame of such phrases Coffey (2009: 237) determines that by far the most frequent initial phrase determiner was indeed indefinite article $a /$ an with token frequency o of 241 out of $380^{4}$. As we see in the Table 1 (Coffey 2009: 237) given below, other determiners with relatively high frequency are demonstratives that and this (the former indicating disapproval and the latter interest, yielding or positive attitude) bearing affective or modal reference. In any case, the meaning of all determiners used in initial position is that of strong evaluative force which can be appreciative or pejorative.

Table 1: Phrase-initial determiners

\begin{tabular}{|c|c|c|c|c|c|c|c|c|c|c|}
\hline a/an & one & the & that & this & \multicolumn{5}{|c|}{ poss.adj poss.noun some sg. ZERO } \\
sg. some pl. ZERO pl.
\end{tabular}

\section{c) Semantic analysis of the pattern a N1+ of +aN2}

The very choice of initial determiners implies the meaning and connotation together with communicative value of the entire pattern itself. In most instances evaluative or descriptive value is distinguished. Although Coffey (Coffey 2009: 240) divides the two, we feel that in most cases descriptive and evaluative aspect intertwine and overlap. However, all instances of this constructional idiom are strongly emotionally charged implying either positive or negative attitude of the speaker, therefore highly subjective. Positive/negative attitude is reflected through the choice of N1 which, consequently, may be more positively or negatively charged and oriented.

3 This may lead to the conclusion that it is the determiner that is crucial for the ultimate interpretation of the constructional idiom.

4 Coffey conducted his study on British National Corpus in which he found total of 7131 examples of the structure a...of a (in the corpus of 250000 words) (Coffey 2009: 230). He analized 380 different expressions of the given pattern. 
Whether appreciative or pejorative (Downing and Locke 2006) in its reference, this construction is always attitudinal, i.e. it expresses the speaker's subjective evaluation of the referent and is interpersonal rather than experiential. That indeed we are dealing with a construction that is semantically anything but neutral is supported by Miladinović's findings (2015:269) which show that intensifier the very occurs relatively often in the determiner position.

(11) He had the name of being the very devil of a fellow.

Whatever the level of syntactic fixedness this constructional idiom may display and irrespective of whether it displays evaluative or descriptive value, clearly, the semantic of the entire construction is rather restricted in the manner that this construction must be emotionally charged, highlighting a subjective attitude of the speaker.

As we are dealing with a productive constructional idiom, whose meaning is quite limited, certain items are much more frequent and more easily collocate within the construction both in $\mathrm{N} 1$ and $\mathrm{N} 2$ position.

Items appearing in the modifying slot (N1) in a larger number of constructs in descending order of frequency are the following: gem (25), giant (20), devil (19), bitch (12), brute (12), monster (11), peach (11), fool (9), bear (8), cracker (8), dream (7) (Coffey 2009: 233).

Semantically, Miladinović (2015: 280-284) ${ }^{5}$ distinguishes the following classes of $\mathrm{N} 1^{6}$ :

1. proper $\mathrm{N}$ [+Animate, + Human] with a positive connotation: an Achilles of $a$, an Apollo of $a$, a Brad Pitt of a, a Casanova of a, a Clark Gable of a, an Einstein of $a$, a Don Juan of a, a Hercules of $a$, a Napoleon of a, a Romeo of $a$, a Zeus of a...

2. proper $\mathrm{N}$ [+Animate, +Human] with a negative reference: $a$ Brutus of a, a Hitler of a, a Macbeth of a, a Mussolini of a, an Othello of $a$...

3. common $\mathrm{N}$ [+Animate, +Human] with a positive reference to people: a baby of $a$, a beauty of $a$, a belter of $a$, a corker of $a, a$ cracker of a, a genius of a, a humdinger of a, a marvel of a, a playboy of $a$, a prodigy of $a$, a stormer of $a$, a stunner of $a . .$.

\footnotetext{
5 Coffey offers similar classification, somewhat less detailed, though. (Coffey, 233)

6 Positive and negative features are not universal to all language communities. it is often the case that the same noun can have both positive and negative reference. Its interpretation is context and culture specific.
} 
Jelena Vujić, Nenad Miladinović: Genitive Constructions as Constructional Idiom in English

3a) Ns denoting mythological and imaginary creatures: an angel of a, a colossus of a, a giant of a, a leprechaun of a, a monster of $a$, a saint of $a$, a wizard of $a . .$.

4. common $\mathrm{N}$ [+Animate, +Human] with a negative reference to people: a barbarian of a, a bastard of a, a bookworm of a, a brute of a, a coward of a, a fool of a, a freak of a, a geek of a, a hag of a, a humbug of $a$, an idiot of $a$, a liar of $a$, a loner of $a$, a midget of $a, a$ rascal of a, a rogue of a, a savage of a, a scoundrel of $a$, a slob of $a$, a smart Alec of a, a sop of a, a swindler of a, a thief of a, a tramp of $a$, a vagabond of $a$, a villain of $a$, a whippersnapper of $a . .$.

4a) Ns denoting mythological and imaginary creatures with negative reference: a devil of $a$, a dickens of $a, a$ dwarf of $a$, a ghost of $a, a$ giant of a, a gnome of $a$, a monster of $a$, a pixie of a, a troll of $a, a$ witch of $a . .$.

5. common $\mathrm{N}$ [+Animate, -Human] with a positive reference to people:

a) $\mathrm{N}$ denoting plants: $a$ daffodil of $a$, a daisy of $a$, a dandelion of $a, a$ flower of $a$, a lily of $a$, an orchid of a, a petunia of $a$, a reed of $a, a$ rose of $a$, a sprout of a, a tulip of $a$, a willow of a...

b) Ns denoting animals: a bird of a, a bull of a, a butterfly of a, a crane of $a$, a dove of a, an eagle of a, a falcon of a, a gazelle of $a$, a hawk of $a$, a lamb of $a$, a lion of $a$, a maverick of $a$, a nightingale of $a$, a tiger of $a$, a wasp of $a$, a whale of $a$, a wolf of $a$...

6. common $\mathrm{N}$ [+Animate, -Human] with a negative reference to people

a) $\mathrm{N}$ denoting plants: a beanpole of a, a tree of $a$, a stick of a, a runner bean of a, a weeping willow of $a .$.

b) Ns denoting animals: an ant of $a$, a bear of $a$, a beast of $a$, a bee of $a$, a bitch of $a$, a bull of $a$, a bulldog of a, a chicken of a, a cow of $a$, a dog of $a$, an elephant of $a$, a frog of a, a giraffe of a, a gnat of a, a goat of a, a goose of a, a hornet of a, a horse of a, a louse of a, a mare of a, a moth of a, a mouse of a, a pig of a, a peacock of a, a rabbit of $a$, a ram of a, a rooster of a, a scorpion of a, a serpent of $a$, , a silly cow of $a$, a snake of $a, a$, a spider of a, a turtle of $a$, a walrus of $a, a$ wasp of a, a whippet of a, a worm of a...

7. Common Ns [-Animate, -Human] with positive reference : $a$ bastion of a, a blockbuster of a, a bulwark of $a$, a dream of a, a heck of $a$, a husk of a, a joy of a, a miracle of $a$, a slab of $a . .$. 
7a) Ns denoting precious stones: a diamond of a, a gem of a, a gemstone of $a$, a jewel of a, a pearl of a, a rough diamond of a...

7b) Ns referring to vehicles and machines: a boneshaker of a, a bulldozer of a, a computer of a, a dynamo of a, a machine of $a$, a Porche of $a$, a rocket of a, a roller coaster of a...

7c) Ns referring to natural phenomena: an ocean of $a$, a rock of $a, a$ whirlpool of a,

7d) Ns referring to food: a cherry of a, a dumpling of a, a honey of $a, a$ peach of $a$, a plum of $a$, chocolate of $a$, a wizard of a...

8. abstract Ns [-Animate] [-Human] with negative reference: an abortion of a, a disaster of $a$, a farce of $a$, a headache of $a$, a hit of $a$, a joke of a, a nightmare of $a$, a pest of a, a ruin of a, a shambles of a, a wreck of $a . .$.

8a) Ns referring to death: a dead duck of a, a grave of a, a graveyard of $a$, a hades of $a$, a hell of a, a morgue of a...

8b) Ns referring to natural phenomena: a mountain of a, a volcano of a, a whirlpool of a,

8c) Ns referring to food : a ham of a, a hamburger of a, a hot dog of a, a mushroom of a, a sausage of a, a tomato of a...

8d) Ns referring to household objects: a hook of a, a trap of a, a ragbag of a, a razor of a, a battleaxe of $a .$.

8e) Ns referring to vehicles and machines: a bulldozer of a, a truck of a, a tank of $a$...

9. Ns denoting taboo words and vulgarisms bearing extremely offensive meaning: an ass of $a$, an asshole of a, a bugger of $a$, a fuck of $a$, a slut of $a$, a sod of a, a shit of $a$, a shit hole of $a$, a whore of $a . .$.

10. common Ns with partitive and attenuating meaning usually with favorable reference: a flicker of a, a glimmer of $a$, a knob of $a, a$ skeleton of a, a stump of a, a wisp of a...

11. common Ns referring to mass, size or quantity: a bundle of $a, a$ heap of a, a hunk of a, a trickle of a, a hulk of a, a lump of a, a scrap of $a . .$.

12. common Ns referring to speed or strength: a blitz of $a$, a lash of $a$, a piledriver of $a$, a scorcher of $a$, a whiplash of $a$, a whizzer of $a . .$.

In addition, Coffey (Coffey 2009: 233) distinguishes a group of miscellanea nouns for which the reference is not quite clearly stated as obviously it can be seen as either positive or negative. In some cases even purely neutral 
Jelena Vujić, Nenad Miladinović: Genitive Constructions as Constructional Idiom in English

only highly descriptive. This group seems to rely on context more than other groups. Nouns that Coffey listed as belonging to this group are the following: bear hug, bolster, bone-shaker, bulwark, chop (movement), colossus, frown, freak, ham (the shape is important), headthumper, iceblockbuster, papyrus, ponytail, razor, scorcher, sexquake, sink, tank-buster, trap, trickle, vocoder, whiplash.

As we can see, in many cases the meaning that the modifier shows a higher or lower degree of (metaphorical) extension. Quite often the positive/negative interpretation will depend on the semantics of N2 it modifies but also on vernacular it is used in and on pragmatic and extralinguistic factors of a particular discourse. For example, in the vernacular of African-Americans the constructs such as a bitch of wheels, a bitch of a bike will be understood as extremely favorable expression describing a particular car or motorbike, while the same modifier in a bitch of a landlady will not be perceived to have a positive reference whatever the social discourse and situational context.

This brings us to the semantic of N2. Coffey (Coffey 2009: 235) notices that the most frequent second nouns are man (73), woman (15), girl and song (8), game (6), goal and thing (5). Other nouns that show relatively high token frequency are day, evening, free kick, job book, doctor, horse, hotel, house, husband, interview, pass (in sport),place, shot (in sport), spider, story, wife, boy, city, clue, cousin, delivery (in sport), dog, father, hill, horseman, match, planet, residence, rush, time, village, voice, wave, year. In most cases N2 has [+Animate, +Human ] reference; the nouns with male reference are more than twice as frequent as those with female reference while the nouns indicating common gender are the least frequent. A wide range of nouns [+Animate+ Human] are found in N2 with man being the most frequent.

Apart from denoting people, which is the most common N2 reference, other notable semantic references of N2 are: a) sport (e.g. 'a freak of a goal'); b) other forms of entertainment (e.g. 'a Godfather of a track'); c) time reference (e.g. 'a bastard of a year'); d) buildings or parts thereof, (e.g. 'that grave of a house'); e) fauna (e.g. 'a beauty of a horse'); f) vehicles and machines (e.g. a monster of a truck).

As noted above the phrase N1ofN2 structurally strongly resembles that of of-genitive phrase and in some cases may be completely identical as in (12a-b). 
(12a) But now Bob was obsessed with the idea that he should get $\underline{a}$ skeleton of a woman and a skeleton of a horse.

(12b) She was a skeleton of a woman whose only desire was to get a two dollar special.

All these semantic features of N1 and N2 create the schema given in (8) which has very few things in common with the genitive meaning as given in (7). Clearly, the two are structurally quite similar but semantically very distinct. Based on what has been said so far, in the following segments of this paper we argue that the pattern $a N 1$ of a N2 is equally syntactic and lexical in its nature. Undoubtedly this is a syntactic word combination that forms a phrasal lexical unit which as such can be characterized in terms of syntactic schema with specific semantic properties (but syntactic as well).

\section{Constructional Morphology approach to the pattern a N1 of a N2 and its interpretation}

The formal, structural and semantic properties of the pattern a N1 of a N2 imply that it shows many phrasal and word-like properties. As such it requires a particular attention and we suggest that it be considered within the framework of $\mathrm{CxG}$, namely $\mathrm{CM}$ which such constructions sees as constructional idioms thus doing justice to the two-faced nature of this phenomenon. Constructional idioms are morphological or syntactic schemas in which one or more positions are lexically fixed (in our case preposition slot is fully lexically fixed, determiner slot is semantically fixed thus lexically semi-fixed) whereas other positions are open slots, represented by variables (Jackendoff 2002).

In the following segments of this paper we will provide arguments for constructional morphology view of of-genitive constructional idioms. We will show that they behave as lexical units in a number of ways.

We have seen that the constructional idiom a N1 of a N2 mainly reflects evaluative and/or descriptive meaning being at the same time strongly emotionally charged. With its constructional properties it qualifies as both a syntactic form and a lexical unit.

Although many regard this kind of construction to be just a marginal phenomenon, which is away from the prototypical genitive construction, 
Jelena Vujić, Nenad Miladinović: Genitive Constructions as Constructional Idiom in English

it can be recognized in some extremely frequent fixed expressions and collocations such as a whale of time, a hell of a..., a heck of a.... Expressions like this are nothing else but highly lexicalized genitive constructional idioms. Due to extremely high level of lexicalization they tend to be perceived as lexical units instead of syntactic units. In perception and conceptualization of such constructs their meaning and lexical nature overshadows their structure.

In retrospect of everything noted before we may say that the construction $\left[[\mathrm{x}]_{\mathrm{Ni}}\left[[\mathrm{of}]_{\mathrm{P}}\left[[\mathrm{a}]_{\mathrm{Det}}[\mathrm{x}]_{\mathrm{Nj}}\right]_{\mathrm{NP}}\right]_{\mathrm{Pp}}\right]_{\mathrm{Nk} \leftrightarrow}\left[\mathrm{SEM}_{\mathrm{j}} \text { with } \mathrm{SEM}_{\mathrm{i}} \text {-like property }\right]_{\mathrm{k}}$ shows both syntactic and word-like lexical unit properties. Its features presented above definitely classify it as a lexical unit or a lexical fixed expression. In order to illustrate the double nature of this construction we argue that the same pattern triggering the same interpretation is found in constructs that are either

a) open idiom structures manifesting fairly low level of fixedness with both $\mathrm{N} 1$ and N2 slots open and only fixed prepositional of form while determiner slots are only categorially and semantically closed (therefore, partially closed). Such is the case with all instances described above as in an angel of a baby, a monster of a truck, this shit of a hotel, etc.

b) proper idioms acting as fixed expressions (closed expression). This same constructional schema triggering the same interpretation is found in some more idiomatic expressions except that they display lexical fixedness and are perceived as conventionalized fixed expressions such as a whale of time, a chit of a girl. They represent invariable syntactic word combinations which are stored in mental lexicon. The speakers perceive them as wholes which refer to particular concepts and are associated with a specific meaning. They are never computed but retrieved. Moreover, the atypical syntactic distribution and ordering of this pattern may be also regarded as idiomatic.

c) semi-open idioms with semi-fixed structure in which the H (N2) is lexically variable and M (N1) is fixed both lexically and semantically with intensifying meaning. Such instances of this construction are a heck of..., a fuck of a.., a hell of a.... .

The classification illustrates the multi-faced nature of this construction which has both lexical and syntactic properties and as such lends itself 
for Construction Morphology. Traditionally, the pattern that is the topic of this paper is seen as a productive syntactic structure that belongs to the syntactic module of language. The idiomatic instances as in b), on the other hand, would be regarded as conventionalized syntactic chunks with a non-compositional semantic interpretation.

Whatever level of fixedness it shows the interpretation and the schema remains the same. What differs though is that they acquire additional communicative and pragmatic value. Therefore, we speak of the constructional idiom which incorporates both phrasal and word-like properties of a N1 of a N2.

We have seen that in this syntactic phrase N2 does not function any longer as a post-modifier of N1 but as a head. At the same time, N1 assumes the role of N2 modifier attributing certain quality to N2. However, the semantic analysis of N1 shows that the words used in N1 position no longer carry their literal meaning as specified in the lexicon but rather a metaphorical meaning with strong emotional charge and intensity. For example, in a dumpling of a woman it is not the woman that has prototypical properties of a dumpling, as a woman can hardly be related to a prototypical property of dumplings. Nouns used in N1 position are actually embedded in the open lexical slot thus acquiring new and quite specific bound meaning, as seen from the given example. The reason for this bound meaning may lie in the fact that N1 is used within a specific bound syntactic environment which affects its meaning. Consequently, for proper reference of the extended $\mathrm{N} 1$ modifying meaning a wider both linguistic and extra-linguistic context is needed like in a Godfather of a track and a godfather of a boss. Actually, it is the extra-linguistic factors that determine the bound meaning of the noun(s) used in N1 slot. Obviously, specific intepretations of all lexical items used in N1 slot are bound to this syntactic construction and they convey a specific modal and emotionally charged meaning only in this immediate syntactic surrounding. This phenomenon is known as heterosemy (Lichtenbeck 1991).

On the other hand, the entire structure has a specific usage and reference of its own and is likely to occur in some contexts rather than in the others. According to Coffey (Coffey 2009: 243) this pattern is found in literature (some 60 examples) but it is more likely to occur in the language of the media and marketing (e.g. a Cracker of a Christmas at Mahnon's). In our pattern $\mathrm{N} 1$ as a modifier has acquired a more general meaning, more abstract and intensive meaning of description and/or evaluation which is 
Jelena Vujić, Nenad Miladinović: Genitive Constructions as Constructional Idiom in English

typically additionally colored with either positive or pejorative meaning as well. In such cases, the meaning of the nouns most commonly used in $\mathrm{N} 1$ position differs from the one when they are used in other syntactic patterns such as regular of-genitive construction or when they are used as independent lexeme.

This naturally suggests that there is a tight semantic relation between $\mathrm{N} 1$ and N2 (N2 restricting the choice on N1) because N2 and N1 cannot be freely selected and combined, which strongly implies that we are truly dealing with the pattern that is more lexical than syntactic in its nature. Furthermore, it is not rare that the meaning of the nouns used as N1 significantly differs when used bound in this construction from the one they may have when used independently. Some nouns may be semantically neutral (e.g. mountain, dumpling) in independent use and then acquire positive meaning in one context and negative in the other, which only shows how all constituents of the pattern (both open and fixed) are mutually interdependent.

(13a) We call him Spud - a spider of a boy, little and quick and a jolly good sort.

(13b) A spider of a woman, hairy, dark, and venomous-looking she had a swollen goiter hanging over the bodice of her dress.

Be it positive or negative reference that N1 conveys, it is nearly always bound to the entire construction and always implies one or the other. Let us look at (13a-b) in which the noun spider has a positive evaluative/ descriptive reference in a) while in b) it has a negative reference when describing a woman. When used independently or in some compounds this noun usually has neutral reference (e.g. spider veins). However, in this of-construction it is anything but semantically neutral in whatever context it appears and whatever interpretation it may trigger.

Idiomatic constructional nature of this pattern is additionally emphasized by the fact that the entire pattern requires a determiner but restricts its choice with a particular meaning. Hence, we are faced with restrictions regarding the choice of determiner- class. The list of frequent determiners given table 1 suggests that all determiners carry similar meaning which in this particular construction is specialized. This is the meaning of a specific reference, indicating " a particular instance, aspect of ...", highlighting the meaning of uniqueness and expressing strong 
approval or dissatisfaction. ${ }^{7}$ This semantic restriction regarding the choice of the determiner also implies the idiomatic nature of the construction which for the reasons of its partial fixedness and idiomatic interpretation can be specified in the constructional idiom of the form given in (8).

In syntactic description of of-genitive constructional idiom we specified that it is not its syntax that deviates from the prototypical English NP but its semantics with the prepositional noun serving as a $\mathrm{H}$ while the default role of a prepositional phrase within an NP is that of a modifier. We refer to this construction as a prepositional head construction which, as noted above, is rather limited to a restricted number of N1and N2 elements. In the framework of CM, by assuming the constructional idiom of the type

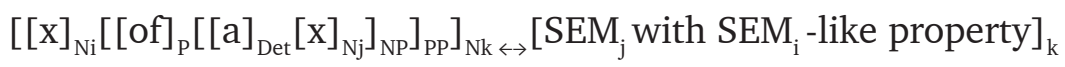

we can analyze prepositional head constructions without losing the generalization that in most English NPs with a prepositional phrase the latter serves as a post-modifier. Our schema representing the constructional idiom at the same time reflects the syntactic properties of the pattern but specifies the differences which distinguish this constructional idiom from a regular possessive of-genitive construction. The given schemas (7) and (8) specify the relation that $\mathrm{N} 1$ and N2 have in both constructions. By using the concept of constructional schema the two syntactic forms receive a clear and straightforward interpretation (Booij 2010: 68). The pattern $a$ N1 of a N2 is not a paragrammatical structure but a constructional idiom. For its atypical syntactic properties our constructional idiom may be called a syntactic nut, which Jackendoff defines as a non-canonical structure of English that is strongly entrenched in the grammar of English, and productive as well (Jackenfoff 2008).

So far we have presented features of the of-genitive constructional idiom which suggests that despite its seemingly clear syntactic structure there is no straightforward syntactic interpretation. Its idiomatic semantic nature shows how it expresses word-like properties which justifies morphological view of such constructions.

Here we provide some arguments for morphological treatment of this particular constructional idiom:

7 If we carefully look into the meanings and references of the determiners used in this construction other than indefinite article $a$ we will notice that none of the determiners has its prototypical meaning. For example, this, that and some have modal affective, emphatic meaning. 
Jelena Vujić, Nenad Miladinović: Genitive Constructions as Constructional Idiom in English

Firstly, the meaning of such constructional idiom often relies on context. The pattern is stored in the lexicon as it is associated with a particular meaning which distinguishes it from the regular computable ofgenitive meaning. Its meaning is more lexeme-like. It is more specific in its semantic reference, not as ambiguous and unclear as genitive (e.g. a portrait of an artist). it is phrasal in structure but not entirely compositional in meaning.

Secondly, we have seen that this undoubtedly (structurally) syntactic construction is semantically parallel to compounds and lexical units in three respects:

a) It is highly (subjectively) descriptive;

b) It has a rather bound meaning that is (although compositional to certain extent) quite conventionalized and not fully cumulative (just as is the case with compounds). It is compositional and conventionalized at the same time. The conventionalized aspect of the meaning of-genitive constructional idiom is expressed as a property of the whole construction.

c) The semantics of N1 and N2 are interdependent and interrelated which is rarely the case with syntactic phrases. On the other hand. the collocability of constituents is rather frequent in compounds.

Lastly, we argue that the meaning of the preposition of in this construction has been strongly lexicalized. The argument for this may be found in the fact that none of the meanings expressed by of-genitive in English can be associated with the meaning of this constructional idiom. Here, the meaning of preposition of is in a way bound to this specific construction.

\section{Conclusion}

The study of the case of-genitive constructional idioms illustrates how languages use complex syntactic patterns to broaden their lexicon (Booij 2010: 191). There is no strict boundary between syntactic and lexical constructs. The pattern a N1 of a N2 illustrates how syntax permeates the lexicon because syntactic units can be lexical. The grammar is a network of syntactic and morphological constructions, with conventionalized instantiations for both types of constructions listed in the lexicon. 
Constructionist approach to syntax, morphology and lexicon can do justice to the fact that large number of phrasal units constituting the lexicon are not words in morphological sense, and yet may form open sets of lexical expressions. Such subsets of complex words with their semantic and functional similarities to simple words are best accounted for through the concept of constructional idioms without giving up the distinction between words and phrases. Construction Morphology operating within Construction Grammar provides an insightful framework for modeling the regularities in the semantic interpretation and formal composition of complex words. In this way our view of the lexicon becomes hierarchical and the neat division between grammar and lexicon ceases to exist. In that respect morphological word-formation operations and syntactic operations function in the same way. In constructionist grammar language production rests on the unification operation of speakers. This means that "well-formed words and sentences are constructed by unifying pieces of information that are specified in the "construction", the list of constructions and simple words of a language" (Booij 2010: 257).

The existence of the type of constructional idiom discussed in this paper across-languages with nearly identical syntactic and semantic properties indicates the strong relation that exists between the two, at the same time opening interesting issues on the cross-linguistic relation that exists between form, meaning and human conceptualization of language structures. Therefore, constructions such as a N1 of a N2 may not be so marginal as they may appear at first sight but require a thorough holistic approach.

\section{References}

Booij, G. (2010). Construction Morphology. Oxford University Press: Oxford. Coffey,S. (2009). A Nightmare Of A Trip, A Gem Of A Hotel: The Study Of An Evaluative And Descriptive Frame. In: Jucker, A. H. et al. Pragmatics And Discourse: Papers From The 29th International Conference On English Language Research On Computerized Corpora (ICAME 29), Ascona, Switzerland, 14-18 May 2008. Amsterdam: Rodopi.

Downing, A. and Locke, P. (2006). English Grammar. A University Course. London: Routledge. 
Jelena Vujić, Nenad Miladinović: Genitive Constructions as Constructional Idiom in English

Huddleston, R. and Pullum, G.K. (2002). The Cambridge Grammar of the English Language. Cambridge: Cambridge University Press.

Jackendoff, R. (2002). Foundations of Language. Oxford: Oxford University Press.

Jackendoff, R. (2008). Construction after construction and its theoretical challenge. Language 84: 8-28.

Lichtenbeck, F. (1991). Language change and heterosemy in grammaticalization. Language 67: 475-509.

Miladinović, N. (2015). Razvoj, strukturalne i semantičke osobenosti sintetičkog $i$ analitičkog genitiva $u$ engleskom jeziku. [Unpublished doctoral dissertation, Faculty of Philology, Univrsity of Belgrade]. 